to the reference assay, clinical sensitivity of the three commercial kits for the detection of resistance was ranging between $89.1 \%$ and $100 \%$ and clinical specificity was ranging between 93.2 and $97.7 \%$, with no statistical significant difference. Testing additional urogenital specimens will allow to specify these percentages.

Conclusion The launch of three sensitive and specific commercial kits for the detection of MG and macrolide resistance will be useful to guide the choice of therapy.

Disclosure No significant relationships.

\section{P610 MYCOPLASMA GENITALIUM IN CLIENTS VISITING TWO DUTCH STI CLINICS: VERY HIGH PREVALENCE AND RESISTANCE TO AZITHROMYCIN}

\begin{abstract}
${ }^{1}$ Sylvia Bruisten*, ${ }^{2}$ David Hetem, ${ }^{3}$ Jean-Marie Brand, ${ }^{4}$ Martijn Van Rooijen, ${ }^{4}$ Paul Oostvogel, ${ }^{4}$ Clarissa Vergunst, ${ }^{2}$ Roel Nijhuis, ${ }^{3}$ Sophie Kuizenga-Wessel, ${ }^{1}$ Alje Van Dam. ${ }^{1}$ Public Health Service Amsterdam, Amsterdam University Medical Center (UMC), Infectious Diseases, Infection and Immunity (AI and II), Amsterdam, Netherlands; ${ }^{2} \mathrm{HMC}$ Westeinde, Medical Microbiology, Den Haag, Netherlands; ${ }^{3}$ Public Health Service Haaglanden, Infectious Diseases, Den Haag, Netherlands; ${ }^{4}$ Public Health Service of Amsterdam, Infectious Diseases, Amsterdam, Netherlands
\end{abstract}

\subsection{6/sextrans-2019-sti.678}

Background Associations between Mycoplasma genitalium $(\mathrm{MG})$ and male urethritis and pelvic inflammatory disease are reported frequently. The recent prevalence of MG among clients of Dutch STI clinics is unknown. Treatment is hampered by resistance to azithromycin.

Methods Urogenital samples from 939 men who have sex with women (MSW), 1025 men who have sex with men (MSM) and 1273 women were included at the STI clinics of Amsterdam and the Hague. Also anal samples from all MSM and from 984 women were included. TMA assays (Aptima, Hologic) were used to detect MG, Chlamydia trachomatis (CT) and Neisseria gonorrhoeae (NG). Azithromycin resistance was tested using a new qPCR with LNA probes and confirmed by 23SrRNA gene sequencing.

Results The overall MG prevalence among STI clients was $19.9 \%$ in MSM, $8.4 \%$ in MSW and $12.5 \%$ in women. Prevalence for all risk groups was higher in STI clients in Amsterdam than in the Hague. MG-positivity was detected in $8.4 \%$ urines of MSW and $6.9 \%$ of MSM. In women, MG was found in $11.0 \%$ of vaginal samples. Of the anal samples of MSM $15.4 \%$ and of women $10.0 \%$ were MG positive. Among MG-positive MSM $67.5 \%$ and among MG-positive women $16.1 \%$ were $M G$-positive in the anal sample only. $M G$ and NG were associated in men $(\mathrm{p}<0.001)$, but not MG and CT. Detected azithromycin resistance was very high: 34/50 (68\%) of urines, 48/81 (59\%) of anal samples and $37 / 75$ (49\%) of vaginal samples.

Conclusion MG is highly prevalent in Dutch STI clinic clients and resistance frequency to azithromycin is very high. In MSM, isolated anal MG is common, but frequently missed if left untested. Anal MG in women is also common, but testing vaginal MG detects most cases. In men, NG and MG infections are associated. Information on clinical relevance of $\mathrm{MG}$ and azithromycin resistance is urgently needed.

Disclosure No significant relationships.
P611 HIGH SEROPREVALENCE OF MYCOPLASMA GENITALIUM IN THE GENERAL ADULT POPULATION OF GERMANY

${ }^{1}$ Felix Reichert*, ${ }^{2}$ Tim Waterboer, ${ }^{3}$ Christina Poethko-Müller, ${ }^{1}$ Klaus Jansen. ${ }^{1}$ Robert Koch Institute, Infectious Disease Epidemiology, Berlin, Germany; ${ }^{2}$ German Cancer Research Center (DKFZ), Infections and Cancer Epidemiology, Heidelberg, Germany; ${ }^{3}$ Robert Koch Institute, Epidemiology and Health Reporting, Berlin, Germany

\subsection{6/sextrans-2019-sti.679}

Background Mycoplasma genitalium (MG) is one of the most common bacterial sexually transmitted infections (STI). Nevertheless, knowledge about the immune response is scarce and seroprevalence has only been assessed in selected populations. In HIV-negative blood donors seroprevalence was 5.5\%, in patients with pelvic inflammatory disease 17\%. We estimated MG seroprevalence in the general adult population in Germany.

Methods We retrospectively analysed sera of the populationbased German National Health Interview and Examination Survey 1998 for MG antibodies using a pre-validated, multiplex, fluorescent bead-based assay. To avoid cross-reactivity, two unconserved regions of adhesion proteins, $\mathrm{MgPaN}$ and $\mathrm{rMgPa}$ were chosen as antigens. The thresholds for seropositivity were set at 1000 median fluorescence intensity units by visual inspection of inflection points. Overall seropositivity for MG was defined as concomitant seropositivity for both antigens. To assure representativeness, survey weights were applied for calculation of prevalence and 95\% confidence intervals (CI). Design-based F was computed as test of independence.

Results Sera of 6038 participants were analysed. Participants were aged 17-79 years (y) (median 44y), 51\% were female. Overall weighted seroprevalence was $6.5 \%$ (95\%CI: $5.7-$ $7.3 \%)$, and by age-group $17-19 y \quad 0.3 \%$ (0.1-1.5\%), 20-24y $1.9 \% \quad(0.9-3.8 \%), \quad 25-29 y \quad 6.7 \% \quad(4.6-9.8 \%), 30-39 y \quad 9.1 \%$ (7.4-11.1\%), 40-49y 10.8\% (8.9-13.0\%), 50-59y 4.5\% (3.4$5.9 \%), \quad 60-69 y \quad 3.3 \% \quad(2.2-5.1 \%)$, and $70-79 y \quad 4.6 \% \quad(2.9-$ $7.4 \%)$. In the age-group $20-29 y$, women had a higher seroprevalence $(7.5 \%, 95 \% \mathrm{CI}: 5.0-11.1 \%)$ than men $(2.1 \%, 95 \% \mathrm{CI}$ : $1.1-4.0 \% ; \mathrm{p}=0.001)$.

Conclusion Compared to other studies, our results for $\mathrm{MG}$ seroprevalence in the general adult population in Germany are plausible.

The earlier increase in women is similar to the pattern in other STI such as Chlamydia trachomatis infection. Seroprevalence dropped in participants aged 50 or more but remained stable at a low level until old age, indicating long antibody persistence or continuous sexual interactions in parts of the population. However, longitudinal studies are necessary to clarify immunological processes.

Disclosure No significant relationships.

\section{P612 ANTIMICROBIAL SUSCEPTIBILITY DATA OF MYCOPLASMA GENITALIUM STRAINS ISOLATED IN JAPAN}

${ }^{1}$ Ryoichi Hamasuna, ${ }^{2}$ Masahiro Matsumoto, ${ }^{2}$ Naohiro Fujimoto, ${ }^{2}$ Tetsuro Matsumoto. ${ }^{1}$ ShinKokura Hospital, Urology, Kitakyushu, Japan; ${ }^{2}$ University of Occupational and Environmental Health, Urology, Kitakyushu, Japan

\subsection{6/sextrans-2019-sti.680}

Background The initial culturing of Mycoplasma genitalium from clinical specimens is still difficult and the antimicrobial susceptibility data of $M$. genitalium strains have not been 
enough. Multidrug resistant $M$. genitalium strains including macrolide or fluoroquinolone-resistance are increasing and analysis of M. genitalium strains is important.

Methods M. genitalium strains were isolated from urinary sediment of M. genitalium-positive urine-specimens from Japanese man. The antimicrobial susceptibility testing was examined by the cell-culture method. The tested antimicrobials were azithromycin (AZM), clarithromycin (CLR), doxycycline (DOX), minocycline (MIN), ciprofloxacin (CIP), levofloxacin (LVX), moxifloxacin (MOX) and sitafloxacin (STFX).

Results Total 14 M. genitalium strains were isolated from Japanese patients. Four strains were isolated in 2003, and other 10 strains were isolated in 2017 and 2018. MIC50 and MIC90 were $\geq 16 \mathrm{mg} / \mathrm{L}$ and $\geq 16 \mathrm{mg} / \mathrm{L}$ for AZM, $\geq 16 \mathrm{mg} / \mathrm{L}$ and $\geq 16 \mathrm{mg} / \mathrm{L}$ for CLR, $.5 \mathrm{mg} / \mathrm{L}$ and $1 \mathrm{mg} / \mathrm{L}$ for DOX, 0.25 $\mathrm{mg} / \mathrm{L}$ and $0.5 \mathrm{mg} / \mathrm{L}$ for $\mathrm{MIN}, 8 \mathrm{mg} / \mathrm{L}$ and $\geq 16 \mathrm{mg} / \mathrm{L}$ for CIP, $4 \mathrm{mg} / \mathrm{L}$ and $\geq 16 \mathrm{mg} / \mathrm{L}$ for $\mathrm{LVX}, 1 \mathrm{mg} / \mathrm{L}$ and $4 \mathrm{mg} / \mathrm{L}$ for MOX and $0.125 \mathrm{mg} / \mathrm{L}$ and $0.5 \mathrm{mg} / \mathrm{L}$ for STFX, respectively. There was no macrolide-resistant strains in 2003, but 9 strains isolated in 2017 and 2018 were resistant to macrolide. Seven strains had high MICs more than $1 \mathrm{mg} / \mathrm{L}$ for MOX, but only one strain had high MIC $1 \mathrm{mg} / \mathrm{L}$ for STFX. Except for one strain which had MIC $2 \mathrm{mg} / \mathrm{L}$ for DOX, MICs of other strains for DOX or MIN were shown between $0.125 \mathrm{mg} / \mathrm{L}$ and $1 \mathrm{mg} / \mathrm{L}$.

Conclusion Among 14 strain, 7 strains had high MICs for macrolide and MOX. In Japan, multidrug-resistant M. genitalium strains were increasing. Limitation of this study was that we tried to isolated M. genitalium strains form patients with treatment-failure cases by macrolide or fluoroquinolone in 2017 and 2018.

Disclosure No significant relationships.

\section{P613 PREVALENCE AND CLINICAL FEATURES OF MYCOPLASMA GENITALIUM IN PATIENTS ATTENDING A STI OUTPATIENT CLINIC IN BERLIN: 2013-2017}

${ }^{1}$ Nathalie Bloeckl, ${ }^{2}$ Slobodan Ruzicic, ${ }^{2}$ Arne Jessen, ${ }^{1}$ Andreas Diefenbach, ${ }^{2}$ Heiko Jessen. ${ }^{1}$ Charité Universitätsmedizin, Berlin, Germany; ${ }^{2}$ Praxis Jessen + Kollegen, Berlin, Germany

\subsection{6/sextrans-2019-sti.681}

Background The Mycoplasma genitalium (MG) infection is a sexually transmitted infection (STI) with often asymptomatic course and increasing antibiotic resistance. One of the risk groups with a high prevalence are men, having sex with men (MSM). To our knowledge we are the first clinic in Germany to test routinely for MG.

Methods We performed a retrospective analysis of all data from MG-tested patients (rectal, pharyngeal and urethral swabs and urine samples) between 2013 and 2017. Due to an absence of consequent test of cure (TOC), an in-depth analysis was performed solely on the samples collected by the first visit of selected patient.

Results A total of 32,302 probes from 7,474 patients were systematically analyzed. Over 5 years we continuously increased testing rates from 3,362 probes (2013) to 11,845 probes (2017). The majority of patients were male (97.0\%), with the mean age of 34.7 years. Most of the patients identified themselves as MSM. Due to patient discomfort, the tests for urethral infection were successively switched from urethral swab to urine probe $(2013,59.6 \%$ vs $2017,88.2 \%)$. The mean prevalence appeared relatively stable and peaked in
2014 (5.2\%). The majority of infections were rectal (6.7\%) and urethral $(4.8 \%)$. Pharyngeal infections were rarely identified $(1.0 \%)$. The urethral swabs appeared as more sensitive when compared to urine probes $(5.5 \%$ vs $4.1 \%)$. A total of $3,819(51.1 \%)$ patients never received a TOC.

Conclusion The presented data represent the largest epidemiological surveillance of MG in Germany to date. The prevalence of MG appeared stable over 5 years. Probably due to many asymptomatic courses the majority of patients did not received a TOC, making them possible vectors in case of treatment failure. Due to increased vulnerability for HIVacquisition in persons with a MG-infection, we recommend routine rectal tests in MSM.

Disclosure No significant relationships.

\section{P614 MACROLIDE RESISTANCE IN MYCOPLASMA GENITALIUM IS STRONGLY ASSOCIATED WITH STI CO- INFECTION}

${ }^{1}$ Martina Furegato*, ${ }^{2}$ Claire Broad, ${ }^{2}$ Laura Phillips, ${ }^{2}$ Emma Heming De-Allie, ${ }^{2}$ Liqing Zhou, ${ }^{1}$ Mark Harrison, 'Sebastian Fuller, 'Emma Harding-Esch, ${ }^{5} \mathrm{~S}$ Tariq Sadiq. ${ }^{1}$ St George's, University of London, Applied Diagnostic Research and Evaluation Unit, Institute for Infection and Immunity, London, UK; ${ }^{2}$ St George's, University of London, Applied Diagnostic Research and Evaluation Unit, London, UK; ${ }^{3}$ St George's, University of London, Applied Diagnostic Research and Evaluation Unit (ADREU), Institute for Infection and Immunity, London, UK

\subsection{6/sextrans-2019-sti.682}

Background Co-infections can compromise empirical therapy when treating genital discharge syndrome (GDS). In the UK, lack of testing for Mycoplasma genitalium (MG), a common cause of GDS, is particularly challenging because of increasing rates of macrolide antimicrobial resistance (AMR). We calculated prevalence of MG co-infections, macrolide resistance and associated risk factors in a diverse symptomatic sexual health clinic (SHC) population.

Methods SHC attendees in England aged $\geq 16$ years, symptomatic of an STI provided: vulvovaginal swabs (females), first void urine (men-who-have-sex-with-women (MSW) and menwho-have-sex-with-men (MSM)), pharyngeal and rectal swabs (MSM). Routine clinic Chlamydia trachomatis (CT)/Neisseria gonorrhoeae (NG) results were obtained and PCR used for MG detection. Macrolide resistance was determined using Sanger sequencing. Unadjusted and risk factor adjusted odds ratios (ORs) for being MG resistant were derived using logistic regression models.

Results Prevalence of MG was 9.5\% across all groups and 6.5\%(95\%CI:4.6-8.9), 12.8\%(9.1-17.3) and 12.3\%(8.5-17.1) in females, MSW and MSM, respectively $(\mathrm{p}<0.005)$. Among patients infected with CT and/or NG, co-infection with $\mathrm{MG}$ was 18.7\%(8.9-32.6), 9.5\%(3.6-19.6) and 4.9\%(1.4-12.2), respectively $(\mathrm{p}<0.05)$. Among MG positives, macrolide resistance was 62.1\%(42.3-79.3), 77.4\%(58.9-90.4), and $90.9 \%$ (70.8-98.9), respectively. In univariate analysis, being MSM (OR:3.0[95\%CI:1.60-5.88]), being of black (3.02[1.66-5.47]) compared to white ethnicity, reported more than one regular partner (3.19[1.25-8.13]), having an STI co-infection (10.13 [4.62-22.25]; $<0.001)$ and a recent STI diagnosis $(2.09[1.18-$ $3.68] ; \mathrm{p}<0.005)$ were associated with having macrolide resistant MG. In multivariable analysis, being MSM (aOR:3.31 [1.44-7.61]), being of black ethnicity (3.31[95\%CI:1.58-6.94]; $\mathrm{p}<0.005)$, more than one regular partner (3.32[1.21-9.08]; 